\title{
Learning potentials and educational challenges of massive open online courses (MOOCs) in lifelong learning
}

\author{
Mie Buhl ${ }^{1}$ - Lars Birch Andreasen ${ }^{2}$
}

Published online: 22 March 2018

(C) Springer Science+Business Media B.V., part of Springer Nature, and UNESCO Institute for Lifelong Learning 2018

\section{Massive open online courses (MOOCs) in a bigger picture}

The MOOC phenomenon contains the potential to draw a large and diverse audience with varying demands of learning possibilities. The characteristics of MOOCs are of interest from a lifelong learning perspective because they offer a possible solution to a rapid and increasing need for education worldwide.

The very first MOOCs were not originally referred to as such; they were only labelled "massive open online courses" (MOOCs) in retrospect, in an attempt to describe what was distinctive and new about the ones which had already been held (Cormier 2008). ${ }^{1}$ These new types of courses explored new ground in many ways. For instance, they explored how to handle a massively large number of participants in a learning environment; or how loose or open the structure of a course could be to still be experienced as a course; or ways of orchestrating various degrees of coherence, connections, collaboration and interaction among participants of a course. These first MOOCs opened up new discussions of pedagogy and didactics and were potentially challenging formerly established ways of organising education and competence development.

\footnotetext{
${ }^{1}$ Cormier, D. (2008). The CCK08 MOOC - Connectivism course, 1/4 way. Dave's Educational Blog [blog post 2 October]. Retrieved 26 January 2018 from http://davecormier.com/edblog/2008/10/02/thecck08-mooc-connectivism-course-14-way/.
}

Mie Buhl

mib@hum.aau.dk

Lars Birch Andreasen

lba@learning.aau.dk

1 Department of Communication and Psychology, Aalborg University, Copenhagen, Denmark

2 Department of Learning and Philosophy, Aalborg University, Copenhagen, Denmark 
Further developments of the MOOC phenomenon have carried it in quite different and sometimes possibly opposing directions. Large internationally recognised universities opened up for everyone interested in access to extensions of their existing courses, which were coined XMOOCs ("x" for extension) and seen as a radically different approach from the original MOOCs, which in parallel were coined cMOOCs (with "c" for connectivity, collaboration, communication, etc.). New companies such as Coursera emerged, which developed global online portals through which universities and other educational institutions or actors could offer courses. Initially, these companies evolved as rapidly expanding frameworks for xMOOC-like courses with standardised ways of structuring, demonstrating and participating. Following this, however, were a widening of the repertoire and additional options for integrating other activities or ways of interaction, meaning that more than only one type of course became available at portals.

Recent developments of MOOCs seem to be driven by commercial interests rather than by pedagogical concerns. MOOC providers are creating new business models which are gradually connecting the educational activities of MOOCs with various degrees of payment. Dhawal Shah $(2017)^{2}$ outlines different business models of four big players on the MOOC market. In his examination of $e d X$, Coursera, FutureLearn and Udacity respectively, he depicts the MOOC phenomenon as becoming more and more based on market mechanisms, where visions about education for everybody are replaced by different payment systems. In his ranking of the four providers, edX represents the most open provider, which still offers some free accessibility, while Udacity was the first to offer a new mode of certification through the so-called Nanodegree in $2014^{3}$ and thereby represents a restricted access to education, and edX succeeded this development of inventing a new university certificate by introducing a modular so-called MicroMasters ${ }^{4}$ programme in 2016 (Young 2016). ${ }^{5}$ Neither of these trademarks, however, are

\footnotetext{
${ }^{2}$ Shah, D. (2017). MOOCs started out completely free. Where are they now? EdSurge [blog post 20 April]. Retrieved 8 January 2018 from https://www.edsurge.com/news/2017-04-20-moocs-started-outcompletely-free-where-are-they-now.

3 "A nanodegree is a course of study which can be completed in less than twelve months and ... the related tuition centres on online learning materials rather than on attendance at some hallowed educational establishment ... The core principle of the nanodegree is that it's vocationally focussed, and not necessarily a one-off qualification but something which people might acquire several of in their working lifetime - or, to use the proposed terminology, a 'stackable' degree" (Maxwell 2015). Maxwell, K. (2015). BuzzWord: Nanodegree. In Macmillan Dictionary [online]. Retrieved 9 February 2018 from https://www.macmillandictionary.com/buzzword/entries/nanodegree.html.

4 A MicroMasters “is the middle ground between a bachelor's degree and a master's degree, and it can save students time and money while satisfying an employer's preference - or requirement - for advanced learning ... MicroMasters programs entail a series of in-depth and rigorous graduate-level courses ... Because these certificates are offered from prestigious universities, the programs are recognized by some of the top companies in the world" (Williams 2017). Williams, T. (2017). MicroMasters: The bridge between a bachelor's degree and a graduate degree: Save time and money while advancing your career. ThoughtCo [blog post 19 November 2017]. Retrieved 9 February 2018 from https://www.thoughtco.com/ micromasters-degree-4149968.

5 Young, J.R. (2016). Why Udacity and edX want to trademark the degrees of the future - And what's at stake for students. EdSurge [blog post 3 November]. Retrieved 16 January 2018 from https://www. edsurge.com/news/2016-11-03-why-udacity-and-edx-want-to-trademark-the-degrees-of-the-future-andwhat-s-at-stake-for-students.
} 
accepted as a formal university degree yet. These examples indicate a general development in some parts of a MOOC movement towards an increasing interest in business and a decreasing interest in discussions of pedagogy. The " $\mathrm{M}$ " for massive and the "O" for open have obtained new meanings.

The idea of openly accessible educational possibilities through which a large number of people can develop competences has also been pursued at national levels, where the concepts of "massive" and "open" take on another meaning. Developing a range of MOOCs covering a national curriculum can be seen as a contribution to solving educational challenges in countries with a lower level of formal education or countries that need a quick educational boost (see for example Nisha and Senthil 2015). ${ }^{6}$

The tendencies mentioned above point to some of the ways in which MOOCs are currently developing towards business and/or socioeconomic interests. From an educational perspective, however, MOOCs are more likely to be regarded as part of the emerging modalities of e-learning, online education, internet connectivity and the use of video and visual resources in relation to learning and competence development.

The bigger picture outlines tendencies of increasing diversity in MOOC offerings in terms of both organisational and educational levels. Current discussions seem to be heading towards the implementation of efficiency strategies which do not necessarily include traditional teaching at formalised educational institutions. In that sense, MOOCs may be the driver for new educational constellations which are potentially emerging outside traditional institutions for educational sciences and pedagogical development.

\section{MOOCs and pedagogy: how are basic pedagogical issues addressed?}

As a newcomer in pedagogical discourses, MOOCs give rise to questions about their pedagogical foundations (see for example Glance et al. 2013; Bayne and Ross 2014). ${ }^{7}$ The development of MOOCs brings up important pedagogical and didactic issues, classical as well as new ones. The aim of this special issue of the International Review of Education - Journal of Lifelong Learning (IRE) is to highlight, within a global perspective of educational systems, some of the learning potentials as well as educational challenges which emerge from the specific development of MOOCs.

MOOCs have developed along with an expansion of the platforms for scholarly discussions. Experiences are reflected, but not only in traditional academic formats

\footnotetext{
${ }^{6}$ Nisha, F. \& Senthil, V. (2015). MOOCs. Journal of Library \& Information Technology, 35(2), 82-89. Retrieved 26 January 2018 from https://search.proquest.com/docview/1672894759?rfr_id=info\%3Axri\% 2Fsid\%3Aprimo.

7 Glance, D.G, Forsey, M., Rieley, M. (2013). The pedagogical foundations of massive open online courses. First Monday, 18(5-6). Retrieved 8 January 2018 from http://firstmonday.org/ojs/index.php/fm/ $\mathrm{rt} /$ printerFriendly/4350/3673.

Bayne, S. \& Ross, J. (2014). The pedagogy of the massive open online course: The UK view. Edinburgh: Edinburgh University.
} 
like scientific journals. Other platforms like business blogs (e.g. Inside higher ed $)^{8}$ are drivers for new insights as well. MOOC pioneers Stephen Downes and George Siemens blogged (e.g. on Stephen Downes, elearnspace or Dave's Educational $B \log )^{9}$ their discussions about the development of the concept on connectivism (e.g. Siemens 2005), ${ }^{10}$ which predicted a new era for higher education. A few years later, Steven Mintz's 2014 blog post on Inside higher ed pointed out that

"Predictions made in 2012 that MOOCs would totally disrupt the existing higher education model were certainly exaggerated. But that does not mean that MOOCs won't have an [sic] profound impact on the future of higher education" (Mintz 2014). ${ }^{11}$

By 2017, blog posts from MOOC students had become important contributions to the ongoing scholarly discussions about where MOOCs are heading. One MOOC student highlights three critical points that MOOC providers need to deal with:

"(a) lack of an effective system to measure and validate the progress of the learners, (b) how to integrate the course credits into the present system so that it counts towards a degree from a college, and (c) how do you ensure personalized guidance and mentorship" (Mehta 2017). ${ }^{12}$

These issues feed into some of the questions this special issue engages with and illuminate the need to discuss how MOOCs operate.

Scholarly discussions about MOOCs seem to fall into two kinds of discourses which are either optimistic or critical towards technology. Not surprisingly, educational studies of the MOOC phenomenon are met with a "scientific toolbox" derived from former developments and insights generated from working with e-learning in a broad sense. These approaches seek to understand the potentials and challenges of MOOCs from e.g. a human agency perspective on human-machine interaction and discuss how technology may promote new learning spaces and change teaching, learning and assessment activities (Xiong and Suen, this issue).

Critical studies of MOOCs' educational prospects may also emerge from other scientific domains outside education which highlight economical, sociological or cultural aspects. These aspects add new perspectives to the investigation of MOOCs and seek to understand the phenomenon in the context of e.g. national economic interests, from a large-scale education perspective, or in terms of the development of a potentially profitable business (Knox, this issue). Socio-cultural perspectives of

\footnotetext{
8 https://www.insidehighered.com/ [accessed 9 February 2018].

9 http://www.downes.ca/; http://www.elearnspace.org/; and http://davecormier.com/edblog/ [all accessed 9 February 2018].

10 Siemens, G. (2005). Connectivism: A learning theory for a digital age. Elearnspace [online article]. Retrieved 26 January 2018 from http://www.elearnspace.org/Articles/connectivism.htm.

11 Mintz, S. (2014). The future of MOOCs. Challenges and lessons. Inside Higher Ed [blog post 29 October]. Retrieved 11 January 2018 from https://www.insidehighered.com/blogs/higher-ed-beta/futuremoocs.

12 Mehta, D. (2017). What according to you is the future of MOOCs? Quora, Forbes [online post 15 March]. Retrieved 11 January 2018 from https:/www.quora.com/What-according-to-you-is-the-futureof-MOOCs/answer/Deepak-Mehta-2.
} 
the digital evolution are used to investigate the implications where human agency is not at the centre of attention (ibid.). Cultural perspectives reveal that the endeavour of globalising education through MOOCs creates paradoxical learning situations when learners are assumed to have similar backgrounds - a misconception caused by cultural blindness towards diverse learning cultures (Andersen et al., this issue).

Alongside theoretical discussions, everyday practices proceed and produce experiences where practitioners engage with and develop different MOOC versions - sometimes by adapting the concept to existing e-learning programmes in open universities (Gil-Jaurena and Domínguez, this issue), and sometimes by applying large-scale technology to a national curriculum and transforming existing formal higher education programmes (Buhl et al., this issue). Experiences from these practices are informing ongoing discussions about technology and learning both in a very concrete sense and at a general level.

Thus, the contributions of this issue jointly represent essential pedagogical questions which are on the one hand specific and action-oriented, and on the other hand feed into overall considerations connected to pedagogical philosophy.

Can the development of MOOCs be pedagogically interpreted as a new addition to existing models for e-learning? Or should technological developments like big data production and calculations by algorithms be followed by a new take on pedagogical philosophy? Is it even possible to discuss pedagogical models with reference to former ideas of learning designs? David George Glance et al. (2013) ${ }^{13}$ argue in favour of regarding MOOCs as an addition to existing models, albeit an addition where the structure and pedagogical foundations are difficult to analyse because they are in some ways close to existing course formats like online courses, or face-to-face teaching or even a blended version of both. Glance et al. (ibid.) see the biggest difference between existing models and MOOCs in the combination of the components and in the massiveness and openness. In other MOOC interpretations, the new combination of components is seen as being transformed into new modes of massiveness and openness by the increasing monetisation of the delivery (e.g. Shah 2017) ${ }^{14}$ and the technologisation of pedagogy (e.g. Knox, this issue).

Positioned between a dehumanisation of facilitating learning processes and a scholarly tradition of exploring and discussing new modes for facilitating learning, this special issue wishes to address the pedagogical implications of implementing MOOCs in lifelong learning systems from a critical perspective, asking:

What are the pedagogical challenges arising from the way MOOCs are practised and assessed, and how do MOOCs engage with different learning cultures, different teaching methods and different learner practices?

The articles presented in this special issue are based on empirical and theoretical discussions from different parts of the world. The purpose of presenting them here is to contribute to the ongoing discussion about MOOCs among scholars and to offer new insights to, for example, educational planners called on to decide which path to take when engaging with MOOCs in lifelong learning systems.

\footnotetext{
13 For bibliographical details, see footnote 7.

14 For bibliographical details, see footnote 2.
} 


\section{The Asia-Europe Meeting (ASEM) and MOOCs}

The work on this special issue took its point of departure in a research network under an international forum, the Asia-Europe Meeting Education and Research Hub for Lifelong Learning (ASEM LLL Hub). ${ }^{15}$ In the research network, which focuses on e-learning in a lifelong learning context, participants from Asian and European countries exchange experiences and collaborate on studies investigating different technology issues from a lifelong learning perspective.

In 2014, the network decided to address the emergence of MOOCs in the current educational landscape by doing national mappings (Kim 2015) ${ }^{16}$ as well as by engaging in a transnational collaborative project. Obviously, the starting points of the participants were very different due to geographic, economic and demographic differences, but also due to diverse theoretical takes on education, on pedagogical traditions and on learning cultures. All of these contributed to drawing a multifaceted picture of how the MOOC phenomenon was received. Questions like "how do we solve the huge challenge of enhancing the education of a fast-growing population?", had an imperative nature for some participants in the network, while others explored how MOOCs formed new aspects of already existing e-learning programmes in formal education systems. MOOCs are claimed to be global. However, the experiences of the network showed that many countries have chosen to engage with MOOCs in a national context. The obvious reason - which is an often overlooked premise in current discourses - is the language of instruction. While the initiators of the global idea of MOOCs are predominantly from English-speaking countries, many countries globally do not have English as a primary language. Another important issue is the implementation of MOOCs. Here some Asian countries are developing alternatives to commercialisation and globalisation efforts, especially American ones, by implementing MOOCs a part of a national education strategy. So far, this seems to have reached its most radical form in India, where MOOCs were implemented in the national higher education curriculum in 2016 (Buhl et al., this issue).

In some parts of the world, governments have initiated national educational programmes based on the MOOC model, aiming to meet a growing demand for education. In other parts, MOOCs have become an element of institutional promotion aiming to attract more students or offer more flexible learning opportunities. All together, the different MOOC practices indicate new teaching and learning patterns which need to be addressed.

\section{The articles}

In order to foster reflections on MOOCs in a lifelong learning perspective, this issue of IRE contributes five articles. The first one is entitled "Beyond the 'c' and the ' $x$ ': Learning with algorithms in massive open online courses (MOOCs)". The author

\footnotetext{
15 For more information about the ASEM LLL Hub, see http://asemlllhub.org/aboutus/ [accessed 13 February 2018].

${ }^{16}$ Kim, B. (Ed.) (2015). MOOCs and educational challenges around Asia and Europe. Seoul: Korea National Open University (KNOU) Press.
} 
Jeremy Knox takes a critical stand towards the emerging role of algorithms in planning and assessing online courses. He argues that the role of technology tends to be overlooked in learning processes and that research so far has merely focused on the construction of knowledge from communicative relations amongst peers, or the behaviour of learners using a particular platform software. He urges that the role of algorithms must also be addressed and he emphasises the importance of realising that algorithms are not to be understood as an external power. Rather, they are socially produced in a mix of human and machine activities. Knox demonstrates how algorithms are already intertwined with MOOCs. He illuminates the ways in which they challenge existing ideas about how learning proceeds. He bases his discussion on three aspects of algorithms which are central to discussions about e-learning: (1) algorithms are not passive arbiters for objective insights; (2) algorithmic processes challenge established theories which locate learning exclusively within or amongst human beings; and (3) algorithms and their implications challenge contemporary education, especially where critical thinking is the aim. Based on these three principles, he identifies and examines four key areas where algorithms hold influence: data capture and discrimination; calculated learners; feedback and entanglement; and learning with algorithms. His line of reasoning leads to a warning - not of the very existence of algorithms, but rather of the lack of transparency in the processes of data production on which learner groups are categorised, teacher decisions are made, and learning is processed. Knox concludes that the well-known division of MOOCs into either cMOOCs or xMOOCs as constructivist and behaviourist positions respectively, is inadequate because it overlooks the role of technology.

In what ways does the development of MOOCs entail new conditions for designing learning processes? In "Upscaling the number of learners, fragmenting the role of teachers: How do massive open online courses (MOOCs) form new conditions for learning design?" Mie Buhl, Lars Birch Andreasen and Karanam Pushpanadham argue that new conditions do indeed apply and that they are characterised especially by a fragmentation of teachers' functions. From a former holistic view of a teacher as the main individual responsible for carrying out the planning, the practical implementation and the reflection, the organigram, as it were, has now changed to a number of fragmented activities being performed by different actors with different areas of responsibility. This article reviews recent studies of new practices and design formats aiming to overcome the upscaling issues of MOOCs. The authors discuss types of MOOCs that integrate inquiry-based and problem-based learning principles with design approaches, or include "authentic assignments", requiring the application of skills and knowledge learned in realworld situations. Many MOOCs are known to have been developed to serve national issues of solving local educational challenges. This is the case in India, for example, where a national initiative develops and provides MOOC courses based on the national curriculum from high school to postgraduate level. A new perspective is that these MOOCs are made mandatory as potential parts of established educational programmes. Through their analysis of the design process of the Indian MOOCs, the authors expose the fragmentation of teacher functions into a hierarchy of actors with different areas of responsibility. The article concludes with the perception by 
Malcolm Knowles et al. (1998) ${ }^{17}$ that a rapport between teacher and learner is crucial in the first place for developing the skills to be self-directed, and that the price for upscaling may be the loss of the teacher as the essential "glue" for connecting the fragments of content, methods and outcome into meaningful situations of learning.

Next, Inés Gil-Jaurena and Daniel Domínguez report on an empirical study they carried out among teaching staff of institutionally driven MOOCs provided by a Spanish distance education university. The authors complement their findings with a theoretical examination of current discussions of conditions for teachers' new roles. In their article entitled "Teachers' roles in the light of massive open online courses (MOOCs): Evolution and challenges in higher distance education", Gil-Jaurena and Domínguez discuss MOOCs as the next step in the evolution of what they refer to as "the open scholarship movement". In accordance with this approach, their empirical study is an examination of how teachers compare teaching a MOOC with teaching a traditional e-learning course in order to reveal the main changes of the teachers' role. Based on characteristics defined by George Veletsianos and Royce Kimmons (2012), ${ }^{18}$ they adopt the notion of open scholarship in order to investigate the broad field of tasks which concern the teacher role and which take three forms: (1) open access and open publishing; (2) open education, including open educational resources and open teaching; and (3) networked participation. Based on these three characteristics, they discuss some of the main problems illuminated by MOOCs whose significant weaknesses are low completion rates, poor quality assurance, a lack of adequate assessment tools, and questions of funding etc. arising in the integration of MOOCs into institutional contexts. The authors' main argument is that learning and assessment modes like adapted learning, collaborative learning and assessment supported by technical tools were already being practised before the advent of MOOCs. The findings from their empirical study comparing ordinary e-learning with MOOCs reveal that MOOCs entail lower teacher presence and interaction with learners, less involvement in assessment, and more involvement in the MOOC's course design than in its practical implementation. Despite these results, the authors conclude that the roles of these teachers had not changed dramatically, since the respondents had already made the transition to digital teaching.

In "Perceptions of authority in a massive open online course: An intercultural study", a group of five authors from three countries discuss cultural aspects which are often overlooked in providers' attempts to offer global education solutions. Drawing on an explorative study of approaches to learning activities in a selected MOOC, Bjarke Linds $\phi$ Andersen, Jaitip Na-songkhla, Cathrine Hasse, Norazah Nordin and Helmi Norman show the different concerns learners have when engaging in a learning environment. Andersen and his co-authors note that questions such as "what impact does it have on the MOOC that participants are situated in

\footnotetext{
${ }^{17}$ Knowles, M. S., Holton, E. F., \& Swanson, R. A. (1998). The adult learner: The definitive classic in adult education and human resource development (5th edn). Houston: Gulf Publishing Company.

18 Veletsianos, G., \& Kimmons, R. (2012). Assumptions and challenges of open scholarship. The International Review of Research in Open and Distance Learning, 13(4), 166-189. Retrieved 19 January 2018 from http://www.irrodl.org/index.php/irrodl/article/view/1313/2304.
} 
diverse cultural contexts?" or "how can we benefit from the differing cultural backgrounds of MOOC participants?" are seldom raised in the research literature on MOOCs. MOOC designers may therefore risk neglecting the cultural diversity of participants. In terms of methodology, the five authors (two from Denmark, two from Malaysia and one from Thailand) all enrolled in a specific MOOC and documented their experiences and the observations they made as online participants. Using an approach called culture contrast, where what is present in one dataset is contrasted with what is absent in another, the authors found that what seems easy for some MOOC participants may present obstacles for others. Andersen et al. argue that the collision of hardware, design and interface of the MOOC with participants' local lifeworlds creates a particular culture which has an important authoritative dimension. It is interesting that this culturally shaped and experienced authority does not exist before the participants inhabit the MOOC. Some proponents of MOOCs tend to assume that participants participate on the same footing and comprise a homogenous "mass". The study of Andersen et al. shows that this assumption is problematic, and potentially gives preference to certain participants from certain cultural lifeworlds over others.

One of the crucial challenges of MOOCs is how to assess students' learning. In "Assessment approaches in massive open online courses: Possibilities, challenges and future directions", Yao Xiong and Hoi K. Suen examine possible ways of assessment that fit open online education. They point out that two kinds of assessment are needed to ensure that genuine learning occurs: assessments for learning (formative assessments), which evaluate students' current progress, and assessments of learning (summative assessments), which record students' cumulative progress. Formative assessment is particularly challenging to conduct within a MOOC, due to the unbalanced instructor-student ratio and the significant variation among the MOOC student population. Summative assessment plays a different role within a MOOC, especially in attempts to integrate MOOCs as course credits into the existing higher education system. This article aims to provide a toolbox for MOOC instructors and instructional designers in devising appropriate assessment for their courses. Xiong and Suen discuss assessment security and analyse various assessment formats such as discussion sessions, automated feedback, multiplechoice quizzes, expert assessment and peer assessment. They point to formative peer assessment as an effective pedagogical strategy through which students may discover alternative approaches to a topic while reading others' work. Peer assessment is also a relevant approach in relation to summative assessment. There are, however, concerns about the accuracy of peer assessment results. Summing up their overview and analyses of the different assessment approaches, Xiong and Suen point out that various ways of peer assessment remain key in the toolbox for assessment in open online education. They recommend optimising the benefits of peer assessment through addition of e.g. small-group peer assessment design, peer rater training, ways of post-hoc (retrospective) corrections of peer rating errors, or by repeating peer assessment during a course, which would enable students to keep revising and developing their work iteratively after acting upon the feedback from peers. 


\section{The promised land: who benefits from MOOCs?}

As an educational newcomer, the MOOC phenomenon prompts optimism as well as scepticism mostly because it is being considered within the discourses it disrupts. As often happens when a new technology emerges, hopes rise that the ultimate education formula has finally arrived. The "massiveness" and the "openness" are intriguing thought patterns in a world with ever more inhabitants, ever more need for education, and an ever-growing need for new solutions for societal and environmental issues. But an exaggerated confidence that e.g. self-directed learners follow learning patterns from well-known constellations by themselves or take up a new constellation - albeit on their own - may lead to misunderstandings of the ways in which "massiveness" and "openness" work for users.

Is it, for example, enough for many MOOC enrollees to participate for their own sake, and to get access to just those resources and those activities in which they participate? Considering the high number of participants who enrol in a MOOC, this might be the case, but does not in a traditional sense carry learners through the whole course. On the other hand, to what extent is the purpose of MOOCs to be equivalent with other kinds of education or competence development? And what demands does this give rise to in relation to which ways of assessment are being used in MOOCs? Is enrolling in a MOOC for example a good "deal" for the learners in the sense of taking a shortcut to a certificate? This could be the case if education is viewed as a ritual rather than as a change of cognitive and social capacity. On the other hand, different learning objectives need different kinds of facilitation, an insight showcased for years in life practices as well as pedagogical practices. Even though MOOCs may not be the promised land, it is important to discuss the implications of MOOCs in all their facets in order to make the best use of them.

\section{The authors}

Mie Buhl is a Professor in Visual Culture, IT and Learning design at Aalborg University (AAU) in Copenhagen, Denmark. Her research revolves around visual learning and knowledge generation in a cross-disciplinary perspective, particularly in relation to the implications of digital technology, social and visual media. She is Director of the Research Center Visual Studies and Learning Design (ViLD) and Head of the knowledge group Communication, IT and learning design (KILD). She is also the coordinator of the Asia-Europe Meeting (ASEM)-network: Development of ICT skills, e-learning and the culture of e-learning in Lifelong Learning. Mie Buhl is an experienced teacher and developer of educational programmes. She has authored/edited more than 100 publications in the research field.

Lars Birch Andreasen is an Associate Professor in Interaction in Virtual Learning Environments at Aalborg University (AAU) in Copenhagen, Denmark. His research deals with collaborative and dialogic learning, social media and new technologies, research methods in social science and humanities, pedagogy in higher education, information literacy, problem-based learning and project work. He is Chair of the coordination group of the Danish cross-university blended Master's programme in ICT and Learning (MIL), Head of the PhD programme of Education, Learning and Philosophy at AAU, and a member of the Asia-Europe Meeting (ASEM)-network: Development of ICT skills, e-learning and the culture of e-learning in Lifelong Learning. 